T. Ono

Nagoya Math. J.

Vol. 92 (1983), 133-144

\title{
ON A GENERALIZATION OF LAPLACE INTEGRALS
}

\author{
TAKASHI ONO
}

\section{Introduction}

Let $\boldsymbol{R}^{n}$ be the Euclidean space of dimension $n \geqq 1$ with the standard inner product $\langle x, y\rangle=\sum x_{i} y_{i}$ and the norm $|x|=\langle x, x\rangle^{1 / 2}, S^{n-1}$ be the unit sphere $\left\{x \in R^{n} ;|x|=1\right\}$ and $d \omega_{n-1}$ be the volume element of $S^{n-1}$ such that $S^{n-1}$ gets the volume 1 . Let $\Omega$ be an open set of $\boldsymbol{R}^{n}$ containing $S^{n-1}$ and let $f: \Omega \rightarrow \boldsymbol{R}^{m}$ be a smooth map. With each integer $\nu \geqq 0$, we shall associate a form $f_{\nu}$ of degree $\nu$ on $R^{m}$ defined by

$$
f_{\nu}(\xi)=\int_{S^{n-1}}\langle\xi, f(x)\rangle^{\nu} d \omega_{n-1}, \quad \xi \in \boldsymbol{R}^{m} .
$$

We then consider the number $\sigma_{\nu}(f)$ which is the mean value of the form $f_{\nu}$ on the sphere $S^{m-1}$ :

$$
\sigma_{\nu}(f)=\int_{S^{m-1}} f_{\nu}(\xi) d \omega_{m-1}, \quad \nu \in Z_{+} .
$$

When $f$ is an affine map: $\boldsymbol{R}^{n} \rightarrow \boldsymbol{R}^{m}$, the function $f_{\nu}$ is substantially the Legendre polynomial of order $\nu$ and $(0.1)$ is the Laplace integral for it $^{11}$. Therefore it is natural to ask questions about forms $f_{\nu}$ associated with more general map $f$.

In this paper, we shall focus our attention on the determination of the number $\sigma_{\nu}(f)$ for any smooth map $f$. It will turn out that the main ingredient of the number $\sigma_{\nu}(f)$ is the number:

$$
N_{k}(f)=\int_{S^{n-1}}|f(x)|^{2 k} d \omega_{n-1}, \quad \nu=2 k^{2)} .
$$

Since $N_{k}(f)=1$ whenever $f$ maps $S^{n-1}$ into $S^{m-1}$, we see that all these "spherical" maps share the same numbers $\sigma_{\nu}(f)$ for all $\nu \in Z_{+}$; hence these numbers measure a deviation of $f$ from being spherical. We shall consider

Received August 23, 1982.

1) See Appendix for a detailed discussion on this matter.

2) As is easily seen, $\sigma_{\nu}(f)=0$ if $\nu$ is odd. 
examples of a family of maps $\left\{f_{p}\right\}_{p \in R}$ for which $\left\{f_{ \pm 1}\right\}$ are Hopf maps and show that the number $N_{k}\left(f_{\rho}\right)$ can be written as a hypergeometric polynomial.

The author would like to mention here that the idea of associating the number like $\sigma_{\nu}(f)$ with a map $f$ came from his earlier work [7] on functions over finite fields.

Notation and conventions

The symbols $Z, \boldsymbol{Q}, \boldsymbol{R}, \boldsymbol{C}$ denote the set of integers, rational numbers, real numbers and complex numbers. The set of non-negative real numbers is denoted by $\boldsymbol{R}_{+}$. We put $\boldsymbol{Z}_{+}=Z \cap \boldsymbol{R}_{+}, \boldsymbol{Q}_{+}=\boldsymbol{Q} \cap \boldsymbol{R}_{+}$. The set of all multi-indices $\alpha=\left(\alpha_{1}, \cdots, \alpha_{n}\right)$ is $Z_{+}^{n}$. We denote by $1_{n}$ the multi-index $(1, \cdots, 1) \in Z_{+}^{n}$. For $\alpha, \beta \in Z_{+}^{n}$ and $x=\left(x_{1}, \cdots, x_{n}\right) \in \boldsymbol{R}^{n},|\alpha|=\alpha_{1}+\cdots+\alpha_{n}$, $\alpha !=\alpha_{1} ! \cdots \alpha_{n} !, m \alpha=\left(m \alpha_{1}, \cdots, m \alpha_{n}\right), m \in Z_{+}, x^{\alpha}=x_{1}^{\alpha_{1}} \cdots x_{n}^{\alpha_{n}}, \alpha \leqq \beta \Leftrightarrow \alpha_{i} \leqq$ $\beta_{i}, 1 \leqq i \leqq n$. For an integer $m, \alpha \equiv 0(m) \Leftrightarrow \alpha=m \beta$ for some $\beta$. When $\beta \leqq \alpha$ we put

$$
\left(\begin{array}{c}
\alpha \\
\beta
\end{array}\right) \stackrel{\text { def }}{=} \frac{\alpha !}{\beta !(\alpha-\beta) !}=\left(\begin{array}{c}
\alpha_{1} \\
\beta_{1}
\end{array}\right) \cdots\left(\begin{array}{c}
\alpha_{n} \\
\beta_{n}
\end{array}\right)
$$

For $a \in C, n \in Z_{+}$we use Appell's notation $(a, n)=a(a+1) \cdots(a+n-1)$ for $n \geqq 1$ and $(a, 0)=1$. For $a, b, c \in C$, the hypergeometric series is defined by

$$
F(a, b ; c ; z)=\sum_{n=0}^{\infty} \frac{(a, n)(b, n)}{(c, n)} \frac{z^{n}}{n !}
$$

For a smooth function $f$ on an open set of $\boldsymbol{R}^{n}$, we put

$$
\operatorname{grad} f=\left(\frac{\partial f}{\partial x_{1}}, \cdots, \frac{\partial f}{\partial x_{n}}\right), \quad \Delta f=\frac{\partial^{2} f}{\partial x_{1}^{2}}+\cdots+\frac{\partial^{2} f}{\partial x_{n}^{2}} .
$$

We shall use the following formulas freely:

$$
\left(x_{1}+\cdots+x_{n}\right)^{\nu}=\sum_{|\alpha|=\nu} \frac{\nu !}{\alpha !} x^{\alpha}, \quad \nu \in Z_{+}, \quad x=\left(x_{1}, \cdots, x_{n}\right) \in \boldsymbol{R}^{n},
$$

$$
\text { when }|\alpha|=2 \nu \text {, we have } \frac{\Delta^{\nu} x^{\alpha}}{\nu !}=\frac{(2 \beta) !}{\beta !}
$$

$$
\text { if } \alpha=2 \beta,=0 \text { if } \alpha \neq 0 \text { (2) . }
$$




\section{$\S 1$. Numbers $b_{\nu}(\ell ; \lambda)$}

Let $\nu \geqq 0, \ell \geqq 1$ be integers and let $\lambda=\left(\lambda_{1}, \cdots, \lambda_{n}\right) \in \boldsymbol{R}^{n}$. We assume that $\ell_{\nu}$ is even: $\ell \nu=2 k, k \in Z_{+}$. We define a number $b_{\nu}(\ell ; \lambda) \in \boldsymbol{Q}_{+}$by

$$
b_{\nu}(\ell ; \lambda)=\frac{\nu !}{k !} \sum_{\substack{|\alpha|=k \\ 2 \alpha \equiv 0(\ell)}} \frac{(2 \alpha) !}{\alpha !(2 \alpha / \ell) !} \lambda^{2 \alpha / \ell} .
$$

If, in particular, $\ell=2$, then $k=\nu$ and

$$
b_{\nu}(2 ; \lambda)=\sum_{|\alpha|=\nu}\left(\begin{array}{c}
2 \alpha \\
\alpha
\end{array}\right) \lambda^{\alpha} .
$$

If, $\lambda=1_{n}$ in (1.2), we have

$$
b_{\nu}\left(2 ; 1_{n}\right)=\sum_{|\alpha|=\nu}\left(\begin{array}{c}
2 \alpha \\
\alpha
\end{array}\right) .
$$

Using the equality

$$
4^{k}\left(\frac{1}{2}, k\right)=k !\left(\begin{array}{c}
2 k \\
k
\end{array}\right)
$$

which one verifies easily, we get the following equality as formal power series in $t$

$$
\sum_{k=0}^{\infty}\left(\begin{array}{c}
2 k \\
k
\end{array}\right) t^{k}=(1-4 t)^{-1 / 2}
$$

and, by (1.2), (1.4), we get

$$
\left.\sum_{\nu=0}^{\infty} b_{\nu}(2 ; \lambda) t^{\nu}=\prod_{i=1}^{n}\left(1-4 \lambda_{i} t\right)^{-1 / 2} \cdot\right]
$$

In particular, we have

$$
\sum_{\nu=0}^{\infty} b_{\nu}\left(2 ; 1_{n}\right) t^{\nu}=(1-4 t)^{-n / 2}
$$

and hence, by (1.3), (1.6), we get

$$
4^{\nu}\left(\frac{n}{2}, \nu\right)=\nu ! b_{\nu}\left(2 ; 1_{n}\right) .
$$

\section{§2. Review of a mean value theorem in potential theory}

Let $\varphi(x)$ be a complex valued smooth function defined on an open set $\Omega$ in $\boldsymbol{R}^{n}$ containing $S^{n-1}$. Assume that either (i) $\Delta^{m} \varphi=0$ for some 
$m \geqq 1$ or (ii) $\Delta \varphi=\lambda \varphi$ for a constant $\lambda \in C$. In this situation, a mean value theorem in potential theory ${ }^{3)}$ tells us that

$$
\int_{S^{n-1}} \varphi(x) d \omega_{n-1}=\sum_{\nu=0}^{\infty} \frac{\left(\Delta^{\nu} \varphi\right)(0)}{4^{\nu} \nu !(n / 2, \nu)}
$$

Needless to say, when $\varphi$ is harmonic, then $m=1$ in (i) or $\lambda=0$ in (ii) and (2.1) is the mean value theorem of Gauss:

$$
\int_{S^{n-1}} \varphi(x) d \omega_{n-1}=\varphi(0) \text {. }
$$

By (1.7), (2.2) can also be written as:

$$
\int_{S^{n-1}} \varphi(x) d \omega_{n-1}=\sum_{\nu=0}^{\infty} \frac{\left(\Delta^{\nu} \varphi\right)(0)}{(\nu !)^{2} b_{\nu}\left(2 ; 1_{n}\right)}
$$

If $\varphi$ is a form of even degree $\ell=2 k$, then $\Delta^{m} \varphi=0$ for $m>k$ and since $\Delta^{m} \varphi(0)=0$ for $m<k$, we get from (2.2)

$$
\int_{S^{n-1}} \varphi(x) d \omega_{n-1}=\frac{\Delta^{k} \varphi}{(k !)^{2} b_{k}\left(2 ; 1_{n}\right)} .
$$

This shows also that

$$
\int_{S^{n-1}} \varphi(x) d \omega_{n-1}=0
$$

if the degree of $\varphi$ is odd, a fact which can be proved directly. On the other hand, in case (ii), we have

$$
\begin{aligned}
\int_{S^{n-1}} \varphi(x) d \omega_{n-1} & =\varphi(0) \sum_{\nu=0}^{\infty} \frac{\lambda^{\nu}}{(\nu !)^{2} b_{\nu}\left(2 ; 1_{n}\right)} \\
& =\varphi(0) \frac{\Gamma(n / 2)}{\left(\sqrt{-\lambda / 2)^{(n-2) / 2}}\right.} J_{(n-2) / 2}(\sqrt{-\lambda})
\end{aligned}
$$

where $J_{\nu}(z)$ is the $\nu$-th Bessel function.

\section{§3. Mean value of quadratic forms}

Let

$$
\varphi(x)=\sum_{|\beta|=2 k} c_{\beta} x^{\beta}
$$

be a form of even degree $2 k$. By (0.5), (2.3), we have

3) See Courant-Hilbert [3], pp. 258-261. 


$$
\int_{S^{n-1}} \varphi(x) d \omega_{n-1}=\frac{(1 / k !) \sum_{|\alpha|=k} c_{2 \alpha}((2 \alpha) ! / \alpha !)}{b_{k}\left(2 ; 1_{n}\right)}
$$

Consider now a diagonal form $f(x)=\lambda_{1} x_{1}^{\ell}+\cdots+\lambda_{n} x_{n}^{\ell}$ and put $\varphi(x)$ $=f(x)^{\nu}$ with $\ell \nu=2 k$. Since we have

$$
\varphi(x)=\sum_{|\sigma|=\nu} \frac{\nu !}{\sigma !} \lambda^{\sigma} x^{\ell \sigma}=\sum_{|\beta|=2 k} c_{\beta} x^{\beta}
$$

where

$$
c_{\beta}=\frac{\nu !}{\sigma !} \lambda^{\sigma} \quad \text { if } \beta=\ell \sigma,=0 \text { if } \beta \not \equiv 0(\ell),
$$

by (3.2), (3.3), we have

$$
\int_{S^{n-1}} f(x)^{\nu} d \omega_{n-1}=\frac{(1 / k !) \sum_{\substack{|\alpha|=k \\ 2 \alpha=\ell \sigma}}(\nu ! / \sigma !) \lambda^{\sigma}((2 \alpha) ! / \alpha !)}{b_{k}\left(2 ; 1_{n}\right)}
$$

From (1.1), (3.4), it follows that

$$
\int_{S^{n-1}}\left(\lambda_{1} x_{1}^{\ell}+\cdots+\lambda_{n} x_{n}^{\ell}\right)^{\nu} d \omega_{n-1}=\frac{b_{\nu}(\ell ; \lambda)}{b_{k}\left(2 ; 1_{n}\right)}, \quad \ell \nu=2 k
$$

If, in particular, $\ell=2$, then $\nu=k$ and we have

$$
\int_{S^{n-1}}\left(\lambda_{1} x_{1}^{2}+\cdots+\lambda_{n} x_{n}^{2}\right)^{\nu} d \omega_{n-1}=\frac{b_{\nu}(2 ; \lambda)}{b_{\nu}\left(2 ; 1_{n}\right)}
$$

Consider a quadratic form $q(x)$ on $\boldsymbol{R}^{n}$ and the integral

$$
\int_{S^{n-1}} q(x)^{\nu} d \omega_{n-1}
$$

Since the change of variable $x \mapsto s x, s \in 0\left(R^{n}\right)$, the orthogonal group, does not change the integral (3.7) and $q(x)$ can be brought to a diagonal form $\lambda_{1} x_{1}^{2}+\cdots+\lambda_{n} x_{n}^{2}$ by such a change of variable, (3.6) implies that

$$
\int_{S^{n-1}} q(x)^{\nu} d \omega_{n-1}=\frac{b_{\nu}(2 ; \lambda)}{b_{\nu}\left(2 ; 1_{n}\right)}, \quad \nu \in Z_{+},
$$

where $\lambda=\left(\lambda_{1}, \cdots, \lambda_{n}\right)$ denotes arbitrarily ordered eigenvalues of $q(x)^{4}$. From (3.8), we get the following equality of formal power series

4) Note that $b_{\nu}(2 ; \lambda)$ is a symmetric function of $\lambda_{i}$ 's. 


$$
\int_{S^{n-1}} \sum_{\nu=0}^{\infty} b_{\nu}\left(2 ; 1_{n}\right) q(x)^{\nu} t^{\nu} d \omega_{n-1}=\sum_{\nu=0}^{\infty} b_{\nu}(2 ; \lambda) t^{\nu} .
$$

Replacing $t$ by $t / 4$ in (3.9) and using (1.5), (1.6), we obtain an interesting equality

$$
\int_{S^{n-1}}(1-t q(x))^{-n / 2} d \omega_{n-1}=\prod_{i=1}^{n}\left(1-\lambda_{i} t\right)^{-1 / 2}
$$

which makes sense if $|t|$ is sufficiently small.

\section{§4. $f_{\nu}(\xi)$ and $\sigma_{\nu}(f)$}

As in Introduction, let $\Omega$ be an open set of $\boldsymbol{R}^{n}$ containing $S^{n-1}$ and let $f: \Omega \rightarrow \boldsymbol{R}^{m}$ be a smooth map. With each $\nu \geqq 0$, we associate a form $f_{\nu}(\xi)$ on $\boldsymbol{R}^{m}$ of degree $\nu$ by

$$
f_{\nu}(\xi)=\int_{S^{n-1}}\langle\xi, f(x)\rangle^{\nu} d \omega_{n-1} .
$$

We shall denote by $\sigma_{\nu}(f)$ the mean value of $f_{\nu}(\xi)$ :

$$
\sigma_{\nu}(f)=\int_{S^{m-1}} f_{\nu}(\xi) d \omega_{m-1} .
$$

To study the numbers $\sigma_{\nu}(f)$ simultaneously for all $\nu \in Z_{+}$, we introduce the generating function

$$
\sigma(f ; t)=\sum_{\nu=0}^{\infty} \sigma_{\nu}(f) \frac{t^{\nu}}{\nu !} .
$$

As is easily seen, the series (4.3) converges for any $t \in C$ and we have

$$
\begin{aligned}
\sigma(f ; t) & =\sum_{\nu=0}^{\infty} \frac{t^{\nu}}{\nu !} \int_{S^{m-1}} d \omega_{m-1} \int_{S^{n-1}}\langle\xi, f(x)\rangle^{\nu} d \omega_{n-1} \\
& =\sum_{\nu=0}^{\infty} \frac{t^{\nu}}{\nu !} \int_{S^{n-1}} d \omega_{n-1} \int_{S^{m-1}}\langle\xi, f(x)\rangle^{\nu} d \omega_{m-1} \\
& =\int_{S^{n-1}} d \omega_{n-1} \int_{S^{m-1}} \sum_{\nu=0}^{\infty} \frac{1}{\nu !}(t\langle\xi, f(x)\rangle)^{\nu} d \omega_{m-1} \\
& =\int_{S^{n-1}} d \omega_{n-1} \int_{S^{m-1}} \exp (t\langle\xi, f(x)\rangle) d \omega_{m-1} .
\end{aligned}
$$

We are thus reduced to compute the integral

$$
\left.\int_{S^{m-1}} \varphi(\xi) d \omega_{m-1} \text { with } \varphi(\xi)=\exp (t<\xi, f(x)\rangle\right) .
$$


A simple computation shows that

$$
\Delta \varphi=\sum_{i=1}^{m} \frac{\partial^{2} \varphi}{\partial \xi_{i}^{2}}=t^{2}|f(x)|^{2} \varphi
$$

Since $\varphi(0)=1$ and $\lambda=t^{2}|f(x)|^{2}$ is a constant for the variable $\xi$, we have, by (2.4),

$$
\int_{S^{m-1}} \varphi(\xi) d \omega_{m-1}=\sum_{k=0}^{\infty} \frac{|f(x)|^{2 k}}{(k !)^{2} b_{k}\left(2 ; 1_{m}\right)} t^{2 k}
$$

and so

$$
\sigma(f ; t)=\sum_{k=0}^{\infty} \frac{N_{k}(f)}{(k !)^{2} b_{k}\left(2 ; 1_{m}\right)} t^{2 k}
$$

with

$$
N_{k}(f)=\int_{S^{n-1}}|f(x)|^{2 k} d \omega_{n-1}
$$

Finally, by (4.3), (4.7), we have

$$
\sigma_{2 k}(f)=\frac{\left(\begin{array}{c}
2 k \\
k
\end{array}\right)}{b_{k}\left(2 ; 1_{m}\right)} N_{k}(f), \quad k \in Z_{+} .
$$

We can also write (4.9) as

$$
\sigma_{2 k}(f)=\frac{b_{k}(2 ; 1)}{b_{k}\left(2 ; 1_{m}\right)} N_{k}(f), \quad k \in Z_{+} .
$$

We shall call a map $f: \Omega \rightarrow \boldsymbol{R}^{m}$ spherical if $f\left(S^{n-1}\right) \subset S^{m-1}$. Since $\left\{f(x) \mid=1, x \in S^{n-1}\right.$, for a spherical map $f$, we have $N_{k}(f)=1$ and so

$$
\sigma_{2 k}(f)=\frac{b_{k}(2 ; 1)}{b_{k}\left(2 ; 1_{m}\right)}
$$

when $f$ is spherical.

\section{§5. Examples}

ExAmple 1. Let $f_{\rho}, \rho \in \boldsymbol{R}$, be the map $\boldsymbol{R}^{2} \rightarrow \boldsymbol{R}^{2}$ defined by $f_{\rho}(x)=$ $\left(x_{1}^{2}-x_{2}^{2}, 2 \rho x_{1} x_{2}\right)$. When $\rho= \pm 1, f_{\rho}$ sends $S^{1}$ onto $S^{1}$; when $\rho=1, f_{\rho}$ is the map $z \rightarrow z^{2}$ of $C=R^{2}$ onto itself and when $\rho=-1, f_{\rho}$ is the map $z \rightarrow \bar{z}^{2}$. Now, 


$$
\left(f_{\rho}\right)_{\nu}(\xi)=\int_{S^{1}} q(x)^{\nu} d \omega_{1}
$$

with $q(x)=\left\langle\xi, f_{\rho}(x)\right\rangle={ }^{t} x A_{\rho} x$ where

$$
A_{\rho}=\left(\begin{array}{cc}
\xi_{1} & \rho \xi_{2} \\
\rho \xi_{2} & -\xi_{1}
\end{array}\right)
$$

whose eigenvalues are $\lambda_{1}=\sqrt{\xi_{1}^{2}+\rho^{2} \xi_{2}^{2}}, \lambda_{2}=-\sqrt{\xi_{1}^{2}+\rho^{2} \xi_{2}^{2}}$. Therefore, by (1.7), (3.8), we have

$$
\int_{S 1} q(x)^{\nu} d \omega_{1}=\frac{b_{\nu}(2 ; \lambda)}{b_{\nu}\left(2 ; 1_{2}\right)}=\frac{b_{\nu}(2 ; \lambda)}{4^{\nu}} .
$$

Since we have

$$
\begin{aligned}
\prod_{i=1}^{2}\left(1-4 \lambda_{i} t\right)^{-1 / 2} & =\left(1-4 \lambda_{1} t\right)^{-1 / 2}\left(1+4 \lambda_{1} t\right)^{-1 / 2}=\left(1-16 \lambda_{1}^{2} t^{2}\right)^{-1 / 2} \\
& =\sum_{k=0}^{\infty}\left(\frac{1}{2}, k\right) \frac{16^{k} \lambda_{1}^{2 k}}{k !} t^{2 k}
\end{aligned}
$$

we get, by (1.5),

$$
b_{2 k}(2 ; \lambda)=\frac{\left(\frac{1}{2}, k\right) 16^{k}}{k !}\left(\xi_{1}^{2}+\rho^{2} \xi_{2}^{2}\right)^{k} .
$$

Or we have

$$
\left(f_{\rho}\right)_{2 k}(\xi)=\int_{S^{1}} q(x)^{2 k} d \omega_{1}=\frac{\left(\frac{1}{2}, k\right)}{k !}\left(\xi_{1}^{2}+\rho^{2} \xi_{2}^{2}\right)^{k}
$$

and

$$
\sigma_{2 k}\left(f_{\rho}\right)=\frac{\left(\frac{1}{2}, k\right)}{k !} \int_{S 1}\left(\xi_{1}^{2}+\rho^{2} \xi_{2}^{2}\right)^{k} d \omega_{1}
$$

Since $\xi_{1}^{2}+\xi_{2}^{2}=1$ on $S^{1}$, we have

$$
\begin{aligned}
\sigma_{2 k}\left(f_{\rho}\right) & =\frac{\left(\frac{1}{2}, k\right)}{k !} \int_{S^{1}}\left(1+\left(\rho^{2}-1\right) \xi_{2}^{2}\right)^{k} d \omega_{1} \\
& =\frac{\left(\frac{1}{2}, k\right)}{k !} \sum_{m=0}^{k}\left(\begin{array}{c}
k \\
m
\end{array}\right)\left(\rho^{2}-1\right)^{m} \int_{S^{1}} \xi_{2}^{2 m} d \omega_{1} .
\end{aligned}
$$

As for the last integral, since the eigenvalues of the quadratic form $\xi_{2}^{2}$ are $\lambda=(0,1)$, we have, by $(1.7),(3.8)$,

$$
\int_{S^{1}} \xi_{2}^{2 m} d \omega_{1}=\frac{b_{m}(2 ;(0,1))}{b_{m}\left(2 ; 1_{2}\right)}=\frac{b_{m}(2 ;(0,1))}{4^{m}}
$$


Since we have

$$
\prod_{i=1}^{2}\left(1-4 \lambda_{i} t\right)^{-1 / 2}=(1-4 t)^{-1 / 2}=\sum_{m=0}^{\infty}\left(\frac{1}{2}, m\right) \frac{4^{m} t^{m}}{m !},
$$

we get, by (1.5),

$$
b_{m}(2 ;(0,1))=\left(\frac{1}{2}, m\right) \frac{4^{m}}{m !}
$$

and

$$
\begin{aligned}
\sigma_{2 k}\left(f_{\rho}\right) & =\frac{\left(\frac{1}{2}, k\right)}{k !} \sum_{m=0}^{k}\left(\begin{array}{c}
k \\
m
\end{array}\right) \frac{\left(\frac{1}{2}, m\right)}{m !}\left(\rho^{2}-1\right)^{m} \\
& =\frac{\left(\frac{1}{2}, k\right)}{k !} F\left(-k, \frac{1}{2} ; 1 ; 1-\rho^{2}\right)
\end{aligned}
$$

because

$$
\left(\begin{array}{c}
k \\
m
\end{array}\right)=(-1)^{m} \frac{(-k, m)}{m !}
$$

Finally, from (4.10) we get

$$
N_{k}\left(f_{\rho}\right)=F\left(-k, \frac{1}{2} ; 1 ; 1-\rho^{2}\right) .
$$

ExAMPLE 2. Let $f_{\rho}, \rho \in R$, be the map $\boldsymbol{R}^{4} \rightarrow \boldsymbol{R}^{3}$ defined by $f_{\rho}(x)=$ $\left(x_{1}^{2}+x_{2}^{2}-x_{3}^{2}-x_{4}^{2}, 2 \rho\left(x_{2} x_{3}-x_{1} x_{4}\right), 2 \rho\left(x_{1} x_{3}+x_{2} x_{4}\right)\right)$. When $\rho= \pm 1, f$ is the classical Hopf map sending $S^{3}$ onto $S^{2}$ (see Hopf [5]). Now,

$$
\left(f_{\rho}\right)_{\nu}(\xi)=\int_{S^{3}} q(x)^{\nu} d \omega_{3}
$$

with $q(x)=\left\langle\xi, f_{\rho}(x)\right\rangle={ }^{t} x A_{\rho} x$ where

$$
A_{\rho}=\left(\begin{array}{cccc}
\xi_{1} & 0 & \rho \xi_{3} & -\rho \xi_{2} \\
0 & \xi_{1} & \rho \xi_{2} & \rho \xi_{3} \\
\rho \xi_{3} & \rho \xi_{2} & -\xi_{1} & 0 \\
-\rho \xi_{2} & \rho \xi_{3} & 0 & -\xi_{1}
\end{array}\right)
$$

whose eigenvalues are

$$
\lambda_{1}=\lambda_{2}=\sqrt{\xi_{1}^{2}+\rho^{2}\left(\xi_{2}^{2}+\xi_{3}^{2}\right)}, \quad \lambda_{3}=\lambda_{4}=-\sqrt{\xi_{1}^{2}+\rho^{2}\left(\xi_{2}^{2}+\xi_{3}^{2}\right)} .
$$

Therefore, by (1.7), (3.8), we have

$$
\int_{S^{3}} q(x)^{\nu} d \omega_{3}=\frac{b_{\nu}(2 ; \lambda)}{b_{\nu}\left(2 ; 1_{4}\right)}=\frac{b_{\nu}(2 ; \lambda)}{4^{\nu}(\nu+1)}
$$


Since we have

$$
\begin{aligned}
\prod_{i=1}^{4}\left(1-4 \lambda_{i} t\right)^{-1 / 2} & =\left(1-4 \lambda_{1} t\right)^{-1}\left(1+4 \lambda_{1} t\right)^{-1}=\left(1-16 \lambda_{1}^{2} t^{2}\right)^{-1} \\
& =1+16 \lambda_{1}^{2} t^{2}+16^{2} \lambda_{1}^{4} t^{4}+\cdots,
\end{aligned}
$$

we get, by (1.5),

$$
b_{2 k}(2 ; \lambda)=16^{k}\left(\xi_{1}^{2}+\rho^{2}\left(\xi_{2}^{2}+\xi_{3}^{2}\right)\right)^{k} .
$$

Or we have

$$
\left(f_{\rho}\right)_{2 k}(\xi)=\int_{S^{3}} q(x)^{2 k} d \omega_{3}=\frac{1}{2 k+1}\left(\xi_{1}^{2}+\rho^{2}\left(\xi_{2}^{2}+\xi_{3}^{2}\right)\right)^{k}
$$

and

$$
\sigma_{2 k}\left(f_{\rho}\right)=\frac{1}{2 k+1} \int_{S^{2}}\left(\xi_{1}^{2}+\rho^{2}\left(\xi_{2}^{2}+\xi_{3}^{2}\right)\right)^{k} d \omega_{2} .
$$

Since $\xi_{1}^{2}+\xi_{2}^{2}+\xi_{3}^{2}=1$ on $S^{2}$, we have

$$
\begin{aligned}
\sigma_{2 k}\left(f_{\rho}\right) & =\frac{1}{2 k+1} \int_{S^{2}}\left(1+\left(\rho^{2}-1\right)\left(\xi_{2}^{2}+\xi_{3}^{2}\right)\right)^{k} d \omega_{2} \\
& =\frac{1}{2 k+1} \sum_{m=0}^{k}\left(\begin{array}{c}
k \\
m
\end{array}\right)\left(\rho^{2}-1\right)^{m} \int_{S^{2}}\left(\xi_{2}^{2}+\xi_{3}^{2}\right)^{m} d \omega_{2} .
\end{aligned}
$$

As for the last integral, since the eigenvalues of the quadratic form $\xi_{2}^{2}+\xi_{3}^{2}$ are $\lambda=(0,1,1)$, we have, by $(1.7),(3.8)$

$$
\int_{S^{2}}\left(\xi_{2}^{2}+\xi_{3}^{2}\right)^{m} d \omega_{2}=\frac{b_{m}(2 ;(0,1,1))}{b_{m}\left(2 ; 1_{3}\right)}=\frac{m !}{4^{m}\left(\frac{3}{2}, m\right)} b_{m}(2 ;(0,1,1)) .
$$

Since we have

$$
\prod_{i=1}^{3}\left(1-4 \lambda_{i} t\right)^{-1 / 2}=(1-4 t)^{-1}=1+4 t+4^{2} t^{2}+\cdots,
$$

we get, by $(1.5), b_{m}(2 ;(0,1,1))=4^{m}$ and

$$
\begin{aligned}
\sigma_{2 k}\left(f_{\rho}\right) & =\frac{1}{2 k+1} \sum_{m=0}^{k}\left(\begin{array}{c}
k \\
m
\end{array}\right) \frac{m !}{\left(\frac{3}{2}, m\right)} \\
& =\frac{1}{2 k+1} F\left(-k, 1 ; \frac{3}{2} ;\left(1-\rho^{2}\right)\right) .
\end{aligned}
$$

Finally, from (4.10) we get

$$
N_{k}\left(f_{\rho}\right)=F\left(-k, 1 ; \frac{3}{2} ;\left(1-\rho^{2}\right)\right) .
$$




\section{Appendix. On Legendre polynomials}

Let $f: \boldsymbol{R}^{n} \rightarrow \boldsymbol{R}^{m}$ be an affine map given by $f(x)=A x+b$ where $A$ is an $(m \times n)$-matrix with real coefficients $a_{i j}, 1 \leqq i \leqq m, 1 \leqq j \leqq n$, and $b$ is a real $m$-vector written vertically. Put $a_{i}=\left(a_{i 1}, \cdots, a_{i n}\right)$, the $i$-th row of $A=\left(a_{i j}\right), M=\left(\left\langle a_{i}, a_{j}\right\rangle\right)$, an $(m \times m)$-symmetric real matrix, and $Q(\xi)$ $={ }^{t} \xi M \xi$, the corresponding quadratic form. We can verify easily that $\lambda=t^{2} Q(\xi)$ satisfies $\Delta \varphi=\lambda \varphi$ for $\varphi=\exp (t\langle\xi, f(x)\rangle)$. Hence, by (2.4), we have

$$
\begin{aligned}
\sum_{\nu=0}^{\infty} f_{\nu}(\xi) \frac{t^{\nu}}{\nu !} & =\int_{S^{n-1}} \varphi(x) d \omega_{n-1} \\
& =\exp (t\langle\xi, b\rangle) \sum_{k=0}^{\infty} \frac{Q(\xi)^{k} t^{2 k}}{4^{k} k !(n / 2, k)}
\end{aligned}
$$

and so

$$
f_{\nu}(\xi)=\sum_{k=0}^{[\nu / 2]} \frac{\nu !}{4^{k} k !(n / 2, k)(\nu-2 k) !} Q(\xi)^{k}\langle\xi, b\rangle^{\nu-2 k} .
$$

Denote by $H$ the algebraic set in $R^{m}$ defined by

$$
H=\left\{\xi \in \boldsymbol{R}^{m} ; Q(\xi)=\langle\xi, b\rangle^{2}-1\right\}
$$

and put $z=\langle\xi, b\rangle$. Then, for $\xi \in H$, we have

$$
\begin{aligned}
f_{\nu}(\xi) & =\sum_{k=0}^{[\nu / 2]} \frac{\nu !}{4^{k} k !(n / 2, k)(\nu-2 k) !}\left(z^{2}-1\right)^{k} z^{\nu-2 k} \\
& =z^{\nu} \sum_{k=0}^{[\nu / 2]} \frac{(-\nu / 2, k)\left(-\nu / 2+\frac{1}{2}, k\right)}{(n / 2, k) k !}\left(\frac{z^{2}-1}{z^{2}}\right)^{k} \\
& =z^{\nu} F\left(-\frac{\nu}{2},-\frac{\nu}{2}+\frac{1}{2} ; \frac{n}{2} ; \frac{z^{2}-1}{z^{2}}\right) \\
& =F\left(-\nu, \nu+n-1 ; \frac{n}{2} ; \frac{1-z}{2}\right) \\
& =P_{\nu, n+1}(z),
\end{aligned}
$$

where the equality between two hypergeometric series follows from a formula of quadratic transformations ${ }^{5}$ and the last equality is a wellknown relation of the Legendre polynomial for $\boldsymbol{R}^{n+1}$ of order $\nu$ and the hypergeometric series ${ }^{6}$. On equating the first and the last terms of (a.3), we get

5) See Magnus-Oberhettinger-Soni [6], p. 50, line 3 from the bottom.

6) See Hochstadt [4], p. 183, line 8. 


$$
P_{\nu, n+1}(\langle\xi, b\rangle)=\int_{S^{n-1}}\langle\xi, A x+b\rangle^{\nu} d \omega_{n-1}, \quad \xi \in H,
$$

which is substantially the Laplace integral for the Legendre polynomials. If, in particular, $m=n+1$,

$$
A=\left(\begin{array}{cccc}
0 & 0 & \cdots & 0 \\
1 & 0 & \cdots & 0 \\
& \cdot & . & 0 \\
0 & 0 & \cdots & 1
\end{array}\right), \quad b=\left(\begin{array}{c}
1 \\
0 \\
\vdots \\
0
\end{array}\right)
$$

and $\xi_{3}=\cdots=\xi_{n+1}=0$, then $\xi_{1}^{2}-\xi_{2}^{2}=1$ for $\xi \in H$ and we get

$$
P_{\nu, n+1}\left(\xi_{1}\right)=\int_{S^{n-1}}\left(\xi_{1}+\sqrt{\xi_{1}^{2}-1} x_{1}\right)^{\nu} d \omega_{n-1},
$$

the Laplace integral in its original form ${ }^{7}$.

\section{REFERENCES}

[1] P. Appell and J. Kampé de Fériet, Fonctions hypergéometriques et hypersphériques, polynomes d'Hermite, Gauthier-Villars, Paris 1926.

[2] B. C. Carlson, Special Functions of Applied Mathematics, Academic Press, New York, 1977.

[ 3 ] R. Courant and D. Hilbert, Methoden der Mathematischen Physik II, 2nd Ed., Springer-Verlag, Berlin Heidelberg New York, 1968.

[4] H. Hochstadt, The Functions of Mathematical Physics, Wiley-Interscience, New York, 1971.

[5] H. Hopf, Über die Abbildungen der dreidimensionalen Sphäre auf die Kugelfläche, Math. Ann., 104 (1931), 637-665.

[6] W. Magnus, F. Oberhettinger and R. P. Soni, Formulas and Theorems for the Special Functions of Mathematical Physics, 3rd Ed., Springer-Verlag, New York, 1966.

[ 7 ] T. Ono, On Certain Numerical Invariants of Mappings over Finite Fields. I., Proc. Japan Acad. Ser. A, 56 (1980), 342-347.

Department of Mathematics

The Johns Hopkins University,

Baltimore, Maryland,

U.S.A.

7) See [4], p. 182, Theorem. 